Relations industrielles

Industrial Relations

\title{
Labour Law under the Charter. Kingston, Ontario, Queen's Law Journal and Industrial Relations Center. 1988, 332 pp.
}

\section{Gilles Trudeau}

Volume 44, numéro 4, 1989

URI : https://id.erudit.org/iderudit/050545ar

DOI : https://doi.org/10.7202/050545ar

Aller au sommaire du numéro

Éditeur(s)

Département des relations industrielles de l'Université Laval

ISSN

0034-379X (imprimé)

1703-8138 (numérique)

Découvrir la revue

Citer ce compte rendu

Trudeau, G. (1989). Compte rendu de [Labour Law under the Charter. Kingston, Ontario, Queen's Law Journal and Industrial Relations Center. 1988, 332 pp.] Relations industrielles / Industrial Relations, 44(4), 953-957.

https://doi.org/10.7202/050545ar

Tous droits réservés @ C Département des relations industrielles de l'Université Laval, 1989
Ce document est protégé par la loi sur le droit d'auteur. L’utilisation des services d'Érudit (y compris la reproduction) est assujettie à sa politique d'utilisation que vous pouvez consulter en ligne.

https://apropos.erudit.org/fr/usagers/politique-dutilisation/ 


\section{RECENSIONS BOOK REVIEWS}

Labour Law Under the Charter, Kingston, Ontario, Queen's Law Journal and Industrial Relations Centre, 1988, 332 pp.

On peut penser qu'au Québec, la controverse au sujet du rapatriement de la Constitution a relégué au second plan la discussion de l'impact d'un autre élément fondamental des décisions politiques en matière constitutionnelle du début des années 80: l'inclusion, dans notre Constitution, d'une Charte des droits et libertés. Cette Charte ${ }^{1}$ reconnaît une large variété de droits généralement appelés droits fondamentaux - et les place au-dessus de l'interférence législative. Dorénavant, il appartient aux tribunaux d'invalider les lois ou autres interventions gouvernementales qui restreignent ou nient les droits et libertés protégés par la Charte.

Malgré la grande importance de la Charte, peu de juristes québécois ont écrit sur le sujet, du moins dans le secteur du droit du travail ${ }^{2}$. La fameuse clause «nonobstant» insérée d'office dans toutes les lois québécoises en $1982^{3}$ n'a certainement pas contribué à sensibiliser le monde québécois des relations du travail à l'impact de la Charte. Nos collègues des autres provinces ont ressenti beaucoup plus rapidement les effets de notre nouvelle Constitution sur l'action législative et gouvernementale de leur juridiction. Pas surprenant qu'ils aient publié davantage sur le sujet ${ }^{4}$.

Aujourd'hui, la Charte canadienne s'applique intégralement au Québec comme ailleurs au Canada. De plus en plus, les citoyens en ressentent les effets dans leur vie quotidienne. Ceci est particulièrement vrai en matière de relations du travail où l'action gouvernementale est omniprésente depuis une cinquantaine d'années. Dans cette perspective, l'ouvrage qu'a publié récemment la Faculté de droit et le Centre de relations industrielles de l'Université Queen's revêt un intérêt considérable pour tous les spécialistes canadiens des relations industrielles.

Cet ouvrage, intitulé Labour Law under the Charter, discute d'un point de vue théorique de l'impact de la Charte sur la législation canadienne concernant le travail. Il regroupe les communications - retravaillées dans la plupart des cas - que plusieurs spécialistes canadiens ont présentées lors d'un colloque tenu sur le sujet à l'Université Queen's en septembre 1987.

1 Charte canadienne des droits et libertés, Partie I de la Loi constitutionnelle de 1982, adoptée comme Annexe B de la Loi de 1982 sur le Canada, R-U, 1982, c.11.

2 L'excellente analyse de Marie-France $\mathrm{BICH}$ fait oeuvre de pionnier dans le domaine: «Petit manuel de guerilla patronale-syndicale: effets de la Charte canadienne des droits et libertés sur le Code du travail», (1987), 47 Revue du Barreau, nº 5, pp. 1097-1179.

3 Loi concernant la Loi constitutionnelle de 1982, L.Q. 1982, c.21. L'effet de la clause «nonobstant» se terminait en avril 1987, puisqu'en vertu de l'article 33 de la Charte, une telle clause ne peut avoir effet au delà de 5 ans sans être renouvelée. Or, la législature québécoise s'est abstenue de renouveler la clause par la suite.

4 Pensons seulement à l'ouvrage de David M. BEATTY, Putting the Charter to Work: Designing a Constitutional Labour Code, McGill-Queen's University Press, 1987, 252 pages. 
Il s'agit d'un débat qui tire ses fondements dans la science politique: en fait tout revient à la souveraineté du législateur et au rôle que doivent jouer les tribunaux dans une démocratie libérale comme la nôtre. Avant 1982, notre système politique, à l'image du régime britannique, reposait sur la souveraineté et la suprématie du législateur constitué de l'ensemble des députés élus par le peuple (à l'exception, bien sûr, des membres du Sénat). L'action législative ne connaissait de limites constitutionnelles qu'à l'égard des frontières juridictionnelles imposées par notre régime fédératif qui répartit les compétences législatives entre deux niveaux de gouvernement. Les tribunaux ne pouvaient invalider une loi à partir de la Constitution canadienne que dans la mesure où le législateur avait agi à l'extérieur de sa compétence constitutionnelle.

Cet ordre de choses a changé en 1982. Dorénavant, l'action législative, comme toute action gouvernementale d'ailleurs, doit se conformer aux prescriptions de la Charte incluse dans notre Constitution nouvellement rapatriée. En conséquence, sera nulle - parce qu'inconstitutionnelle - toute loi qui contrevient à une disposition de la Charte. Et ce sont les tribunaux qui, à la demande d'une partie, réviseront l'action législative et gouvernementale pour s'assurer de sa conformité à la Charte. Une loi qui nie ou limite un droit ou une liberté protégée par la Constitution sera nécessairement invalidée par les tribunaux ${ }^{5}$. Dans cette perspective, la nouvelle Constitution confère un important pouvoir de contrôle et de révision de l'action législative aux tribunaux.

Ce pouvoir est d'autant plus important que la rédaction de la Charte est large et donne ouverture à beaucoup d'interprétation. Ainsi jusqu'où s'étend la portée des libertés reconnues comme celles d'expression, de réunion, et d'association. Dans chaque cas, ce sont les tribunaux qui devront en décider en définissant le contenu de ces libertés fondamentales. Par ailleurs, l'article 1 de la Charte permet de restreindre un droit ou une liberté protégés «dans des limites qui soient raisonnables et dont la justification puisse se démontrer dans le cadre d'une société libre et démocratique». Plusieurs voient dans cette dernière disposition une invitation faite aux tribunaux d'exercer une fonction qui devrait appartenir en exclusivité au législateur dans une société démocratique.

En effet, il ne faut pas perdre de vue que, dans notre régime politique, les membres de la magistrature ne sont pas élus mais nommés par le gouvernement en place. La Charte permet donc à des individus non élus de réviser l'action législative des représentants du peuple à partir de règles formulées très largement dont l'interprétation laisse obligatoirement place à une grande discrétion personnelle.

Voilà très sommairement résumé le fond du débat que le lecteur retrouvera dans l'ouvrage publié par l'université Queen's. Cette discussion se situe, comme le titre l'indique, dans le cadre du droit du travail et des relations industrielles. Ce secteur du droit offre une emprise particulière aux dispositions de la Charte. En effet, comme nous le mentionnions plus haut, il s'est développé à partir d'une intervention importante du législateur qui a généralement consacré la préséance des droits de la majorité sur ceux des individus. Pensons seulement au concept de monopole de représentation syndicale et à l'accréditation qui le consacre. Comment le contenu de notre droit du travail résistera-t-il à une révision judiciaire faite à la lumière de la Charte? Le mouvement ouvrier bénéficiera-t-il d'une telle révision?

5 Dans la mesure bien sûr où le tribunal décide que cette négation ou cette limite n'est pas justifiée dans le cadre d'une société libre et démocratique selon les termes de l'article 1 de la Charte. Le législateur peut aussi écarter temporairement l'application d'une partie importante de la Charte en utilisant la clause «nonobstant» conformément à l'article 33. 
Plusieurs auteurs dont la réputation de la plupart n'est pas à faire donnent leur opinion. Leur discussion est généralement très théorique. Des considérations les plus variées guident leur raisonnement. Tous réfèrent à des degrés divers à la trilogie de la Cour suprême du Canada qui refuse d'étendre la protection constitutionnelle accordée à la liberté d'association à la négociation collective et au droit de grève ${ }^{6}$.

Comme on pouvait s'y attendre, les opinions divergent passablement d'un auteur à l'autre. Ainsi, des enseignements fort différents sont tirés de la trilogie de la Cour suprême selon celui qui l'analyse. Le professeur Bernard Adell, dans le chapitre d'introduction qu'il signe, divise les auteurs en deux catégories: les «sceptiques» et les «idéalistes».

Les «sceptiques» entretiennent peu d'espoir quant aux conséquences de l'application de la Charte canadienne au droit du travail: selon eux, le mouvement ouvrier et les travailleurs en général seront laissés pour compte dans ce processus. Ces auteurs doutent de la capacité des tribunaux d'appliquer les principes de la Charte de façon objective, à l'abri des pressions externes, particulièrement celles provenant de la classe dominante.

À l'inverse, les «idéalistes» croient les tribunaux capables d'utiliser des arguments de principe et de rendre des décisions qui favoriseront le progrès social. Ainsi, ce que le professeur Adell appelle la persuasion rationnelle fondée sur la Charte pourrait se substituer au pouvoir collectif des travailleurs. Les plus démunis de la classe ouvrière pourraient ainsi tirer grand profit de cette substitution.

Il serait vain et fastidieux de tenter ici de résumer systématiquement l'opinion de chaque auteur. Contentons-nous plutôt de souligner quelques-uns de leurs arguments les plus percutants.

Ainsi, Harry W. Arthurs est fort pessimiste quant aux effets de la Charte sur le droit du travail. Historiquement, les tribunaux ont toujours eu une attitude négative à l'endroit du mouvement ouvrier. Laisser à ces mêmes tribunaux le soin d'interpréter une Charte conçue avant tout pour protéger des droits individuels ne peut être que néfaste au mouvement ouvrier. Arthurs soutient cependant que puisque le droit n'affecte pas beaucoup la vie quotidienne, l'avènement de la Charte ne modifiera pas de façon substantielle la pratique des relations du travail. La nouvelle Constitution aura par ailleurs des effets indirects importants, notamment en provoquant un changement dans la culture légale qui marginalisera le droit du travail. Cette nouvelle culture favorise l'intervention des juges et le développement du litige judiciaire au détriment du fonctionnement des institutions spécifiques aux relations du travail comme les conflits industriels et l'arbitrage.

Le même pessimisme est partagé par Thomas Kuttner qui illustre comment la négociation collective est exclue du giron constitutionnel. Ceci contraste avec l'époque antérieure à la Charte où la suprématie du Parlement avait suffi à assurer la reconnaissance judiciaire de la négociation collective. Sans protection constitutionnelle, le développement de la négociation ne sera plus assuré aussi efficacement par les commissions des relations du travail. En effet, leurs décisions seront de plus en plus sujettes à révision par des tribunaux appliquant les valeurs transmises par la Charte, valeurs étrangères à celles à la base de la négociation collective.

6 Cette trilogie est constituée des décisions suivantes: Re Public Service Employee Relations Act, [1987] 1 R.C.S. 313; P.S.A.C. c. Canada, [1987] 1 R.C.S. 424; R.W.D.S.U. c. Saskatchewan, [1987] 1 R.C.S. 460. 
Judy Fudge démontre à quel point les questions auxquelles les tribunaux doivent répondre à partir de la Charte sont de nature politique. Ainsi en est-il de la détermination du champ d'application de la Charte (définition d'action gouvernementale), de la définition des droits et libertés qu'elle protège et de la portée de l'exception de son article 1. La réponse à ces questions impose aux tribunaux l'utilisation de critères subjectifs. L'auteure effectue aussi une revue détaillée des décisions des tribunaux et organismes juridictionnels pour analyser la façon dont est appliquée l'exception de l'article 1 de la Charte en matière de relations de travail. Au terme de cet exercice, elle conclut que vraisemblablement, les tribunaux n'utiliseront pas la Charte de façon à favoriser les intérêts de la classe ouvrière.

Geoffrey England affirme que même si la Cour suprême avait étendu la protection constitutionnelle à la négociation collective et au droit de grève, le mouvement ouvrier n'en aurait pas tiré beaucoup d'avantages. En effet, cette "constitutionnalisation» du droit de grève n'aurait pas pour autant changé le contenu de la réglementation actuelle en matière de relations du travail que l'auteur perçoit comme un outil de récupération de l'énergie du mouvement ouvrier. Les changements nécessités pour faire de la négociation un véritable promoteur de justice sociale doivent nécessairement provenir du législateur, non des tribunaux. Dans cette perspective, la Charte est incapable de produire de tels changements, qu'elle reconnaisse ou pas le droit de négociation et de grève.

Paul Cavaluzzo rejoint l'opinion d'England dans la mesure où il affirme aussi que le partage des intérêts en matière de politique de relations du travail relève de la législature et non des tribunaux. Dans cette perspective, il se réjouit de la trilogie de la Cour suprême concernant le droit de grève qui forcera le mouvement ouvrier à rechercher dans l'arène politique plutôt que judiciaire la reconnaissance de ses droits.

À l'opposé, le courant idéaliste est représenté principalement par David Beatty et Steve Kennett. Selon eux, les catégories de travailleurs moins bien organisés et représentés dans notre société pourront utiliser la Charte et les tribunaux pour revendiquer la part qui leur revient et qu'ils n'ont pas réussi à obtenir auprès du législateur. Beatty et Kennett reprennent les principaux arguments de leurs détracteurs pour en démontrer le caractère fallacieux. Ils affirment que les tribunaux possèdent les outils leur permettant d'interpréter de façon rationnelle et objective la Charte. Dans cette perspective, les auteurs avancent que la décision de la Cour suprême qui nie à la négociation collective et au droit de grève la protection constitutionnelle est erronée. Une argumentation logique et serrée aurait amené la Cour à une conclusion contraire.

Brian Langille s'inscrit aussi dans le courant idéaliste. Dans son long exposé, il s'applique presqu'exclusivement à réfuter les arguments de plusieurs constitutionnalistes canadiens qui mettent en doute la capacité du droit de limiter et de diriger l'intervention judiciaire. Cette incapacité proviendrait essentiellement de la nature intrinsèquement indéterminée du langage qui est le médium utilisé par le droit pour contenir l'activité judiciaire. Ainsi, le juge pourrait conférer la signification que lui dictent ses valeurs personnelles aux mots utilisés dans la loi. Langille soutient que cette affirmation à la base du courant sceptique provient d'une lecture erronée des écrits du philosophe Wittgenstein et qu'en conséquence, il est mal fondé.

L'Université Queen's nous offre donc un ouvrage dans lequel les idées s'affrontent. Cette caractéristique est d'autant plus intéressante que le sujet traité est extrêmement controversé. Dans ce domaine, le lecteur est beaucoup mieux servi par un tel affrontement que par la présentation d'une opinion monolithique.

On ne peut rester indifférent à la lecture de l'ouvrage. Que ses effets soient bénéfiques ou non pour le mouvement ouvrier, que son application favorise le statu quo ou le progrès social, 
il demeure que la Charte aura un impact sur le droit du travail et les relations industrielles. Cet impact s'est d'ailleurs déjà manifesté, que ce soit seulement par le nombre d'instances où elle est invoquée. Ce seul phénomène est très important en lui-mềme, comme le note le professeur Arthurs.

Toute personne intéressée au droit du travail devrait prendre connaissance de cet ouvrage. Il révèle une dimension nouvelle et, pour plusieurs, inconnue de l'analyse dans ce domaine. C'est un ouvrage avant tout théorique qui tient autant des sciences politiques et de la philosophie que du droit. Il nous fournit un bel exemple d'une étude «on law» plutôt que «in law», selon l'expression de Bernard Adell'. Le lecteur du Québec constatera l'avancement de nos collègues du Canada anglais dans ce type d'étude par rapport à la recherche québécoise.

Par ailleurs, l'ouvrage présente les lacunes communes à tout collectif. On y note un manque d'uniformité dans l'analyse. Certains textes sont trop longs, d'autres un peu superficiels. Des répétitions inutiles se produisent d'un texte à l'autre, notamment dans l'analyse que certains auteurs font de la trilogie de la Cour suprême. On doit savoir gré à Bernard Adell qui a décelé un fil conducteur entre tous ces textes et proposé une introduction à leur lecture. Seul le texte de George Adams fait davantage bande à part, traitant des pouvoirs dont disposent les tribunaux face à une loi qui contrevient en tout ou en partie à la Charte. Bien qu'intéressante en soi, cette analyse ne s'inscrit pas dans la trame qui lie les autres textes ensemble.

De façon générale, l'ouvrage se lit bien. Les éditeurs ont fait un excellent travail. La présentation est fort acceptable et constante d'un texte à l'autre. Les auteurs ont su présenter leur position de façon claire et accessible à l'ensemble des lecteurs intéressés au domaine. Seul le texte de Brian Langille affiche des difficultés telles qu'il rebutera le lecteur non initié à la philosophie nord-américaine du droit.

Somme toute, malgré certaines lacunes, le livre publié par l'Université Queen's fait oeuvre utile. Il sensibilise le lecteur à une nouvelle dimension - combien fondamentale - de notre droit du travail. En fait, tout intéressé aux relations industrielles en général, et au droit du travail en particulier, devrait prendre connaissance du débat présenté dans ce livre. Cet exercice ne le laissera pas indifférent.

Gilles TRUDEAU

Université de Montréal

Labour Relations: An Experiential and Case Approach, by Roger Wolters and William H. Holley, New York, The Dryden Press, 1988, 321 pp., ISBN 0-03-069304-7

Despite the decline in industrial relations enrolments in the United States, new textbooks appear frequently, many with innovative features. This book is distinguishable from other labour relations textbooks by combining introductory discussions of principles typically found in texts with a large number of cases. Topics covered include the major labour relations problems confronted by the practitioner: the acquisition of bargaining rights by a union, negotia-

7 Bernard ADELL, «Le droit et les relations industrielles dans les juridictions canadiennes de Common Law», dans L'état de la discipline en relations industrielles au Canada, sous la direction de G. HÉBERT, H.C. JAIN et N.M. MELTZ, Monographie $\mathrm{n}^{\circ} 19$, Montréal, Ecole de relations industrielles, Université de Montréal, 1988, pp. 131 à 180. 